\title{
The Molecular Structure of Microtubule-Associated Protein 1A (MAP1A) in vivo and in vitro. An Immunoelectron Microscopy and Quick-Freeze, Deep-Etch Study
}

\author{
Yoko Shiomura and Nobutaka Hirokawa \\ Department of Anatomy, School of Medicine, University of Tokyo, Hongo, Tokyo, Japan, 113
}

We studied the distribution of microtubule-associated pro-
tein 1A (MAP1A) in Purkinje cell dendrites by means of elec-
tronmicroscopic immunocytochemistry, using a monoclonal
antibody (McAb) against MAP1A; this was combined with
the observation of the 3-dimensional cytoskeletal ultrastructure in dendrites via the quick-freeze, deep-etch technique (QF-DE). We prepared a McAb against rat brain MAP1. This MCAb recognized MAP1A on a nitrocellulose filter through use of the immunoblotting method, and stained immunofluorescently Purkinje cell perikarya, dendrites, and axons. Using the McAb, we labeled rat cerebellum extracted with Triton $X-100$ and simultaneously fixed with aldehyde, followed by gold-labeled rabbit anti-mouse IgG. Gold particles were attached to the filamentous, fuzzy materials, mostly those connected to microtubules (MTs), but were hardly localized on those attached to neurofilaments (NFs). The 3-dimensional cytoskeletal ultrastructure of fresh Purkinje cell dendrites was revealed by QF-DE. In Purkinje cell dendrites, MT was a predominant cytoskeletal element, whereas only a few NFs were found. Fine, elaborate cross-bridges filled up the interstices among MTs, and between MTs and other cellular components. Cross-bridges linking MTs to one another were composed mainly of a fine filamentous structure, frequently branching and anastomosing at several sites, and appeared somewhat granular. We ensured that the crossbridges observed in saponin-extracted tissues were not a result of artifactual condensations or precipitations of soluble proteins during deep etching.

The molecular structure of MAP1A was further investigated by the rotary shadowing technique. The affinity-purified MAP1A was a long, thin, filamentous, and very flexible molecule. We also studied taxol-stabilized MTs decorated with MAP1A by means of QF-DE. It was shown that MAP1A extended outward from the MT wall as thin filaments connecting MTs to one another. These observations have led to the conclusion that MAP1A is possibly one of the main components of the cross-bridges between MTs in Purkinje cell dendrites.

\footnotetext{
Received July 14, 1986; revised Nov. 19, 1986; accepted Dec. 15, 1986.

The authors wish to express their appreciation for the valuable advice and technical guidance regarding the monoclonal antibody method to Drs. Tomio Tada and Ryo Abe, Department of Immunology, University of Tokyo; Yasuo Ihara, Tokyo Metropolitan Institute of Gerontology; and Takeshi Tanaka, Japan Red Cross IIospital, Saitama Branch. The authors are also grateful to Ms. Y. Kawasaki and Mr. Y. Fukuda for their technical assistance. The present study was supported by grants-in-aid from the Ministry of Education, Science and Culture of Japan and from the Muscular Dystrophy $\Lambda$ ssociation of America to N.H

Correspondence should be addressed to Yoko Shiomura at the above address. Copyright (C) 1987 Society for Neuroscience $0270-6474 / 87 / 051461-09 \$ 02.00 / 0$
}

Neurons are highly polarized cells; that is, they receive stimuli mainly at synaptic sites on dendrites and soma, integrate the stimuli at the dendrites and soma, and initiate and conduct these impulses through the axons. They develop characteristic 3-dimensional structures for this purpose. In this regard, the differentiation of the cytoskeletal organization can be considered as providing a necessary base for the various functions within the nerve cells. It is well known that microtubules (MTs) and neurofilaments (NFs) are basic cytoskeletal elements in nerve cells. In order to understand the differentiated functions in neurons, a prerequisite is the study of both the cytoskeletal structure and the distributions of the chemical components of each part of these cells.

Recently, extensive biochemical and immunofluorescent studies have been carried out in order to characterize these cytoskeletal components and their associated proteins. The microtubule-associated proteins (MAPs) from brain tissue have becn charactcrized and categorized into several classes: highmolecular weight-proteins $\left(\mathrm{MAP} 1, M_{\mathrm{r}}=350,000 ; \mathrm{MAP} 2, M_{\mathrm{r}}=\right.$ 270,000) (Borisy et al., 1975; Sloboda et al., 1975), a family of closely related lower-molecular-weight proteins ("tau," $M_{\mathrm{r}}=$ 55,000-70,000) (Weingarten et al., 1975; Cleveland et al., 1977a, b), and low-molecular-weight MAPs (Vallee and Davis, 1983). Among these, MAP1 and MAP2 are the most prominent nontubulin proteins in brain MT preparations. Purified MAP1 (Vallee and Davis, 1983) and MAP2 (Herzog and Weber, 1978; Kim et al., 1979; Gottlieb and Murphy, 1985) have the appearance in vitro of regularly spaced filamentous arms on the MT surface. MAP1 has been further categorized into at least 3 subspecies: MAP1A, MAP1B, and MAP1C (Bloom et al., 1984). Tau induces the formation of rather smooth MTs, without the arm expression (Weingarten et al., 1975; Herzog and Weber, 1978). Tubulin itself makes up the structural backbone of the MT. These MAPs show a characteristic subcellular distribution in neurons. MAP1 is distributed widely in various tissues and in both dendritic and axonal processes of neurons (Matus et al., 1983; Bloom et al., 1984; Huber and Matus, 1984). However, MAP2 is preferentially localized in the dendritic processes of neurons (Miller et al., 1982; Matus et al., 1983; DeCamilli et al., 1984), and tau is predominantly detected in axons (Binder et al., 1984).

Until now, little has been known about how these proteins organize in vivo the cytoskeletal architecture at the ultrastructure level. MAP2 has been considered to be associated with intermediate filaments in cultured brain cells (Bloom and Vallee, 1983) and NFs in $\beta, \beta^{\prime}$-iminodipropionitrile (IDPN)-intoxicated rat axons (Papasozomenos et al., 1985), while MAP1A and 1B 
have been reported to be components of cross-bridges between MTs in IDPN-intoxicated rat axons (Hirokawa et al., 1985).

Purkinje cells in the cerebellar cortex develop characteristic dendrites, which have been favorite targets of investigations into the relationship between MTs and MAPs in neuronal tissue in vivo. However, the cytoskeletal architecture of dendrites has not been well understood, compared with that of axons (Hirokawa, 1982; Schnapp and Reese, 1982; Hirokawa et al., 1984, 1985; Hirokawa and Yorifuji, 1986). Furthermore, it has not as yet been determined what functions these proteins perform in the construction of the cytoskeletal architecture in dendrites at the molecular level, although extensive immunofluorescent studies have been carried out on both MAP1 and MAP2 .

In the present investigation we carried out immunoelectronmicroscopic studies using our anti-MAP1A monoclonal antibody $(\mathrm{McAb})$ combined with the quick-freeze, deep-etch technique (QF-DE) on rat Purkinje cell dendrites. At the same time we studied the molecular structure of affinity-purified MAP1A and the taxol-stabilized MTs decorated with MAP1A. We found that dendrites consist of MTs and extensive networks of crossbridges and that MAP1A is a long, thin, rodlike structure that is a major component of these cross-bridges between MTs in the dendrites.

\section{Materials and Methods}

Preparation of monoclonal antibody against MAP1A. We prepared a $\mathrm{McAb}$ against rat brain MAP1A. MAP1 was purified as an immunogen by the following method. First, rat brain MT proteins were isolated by the taxol procedure (Vallee, 1982) and subjected to preparative SDSPAGE on a $7 \%$ polyacrylamide gel (Laemmli, 1970). Then the MAP1 bands were excised from the gel and homogenized in $0.01 \mathrm{M}$ phosphate buffer ( $\mathrm{pH}$ 7.4). Homogenates containing MAP1 were stirred in the phosphate buffer with SDS (final concentration, $5 \%$ ) overnight at room temperature in order to elute the protein from the gel, and then centrifuged at $10,000 \mathrm{rpm}$ for $10 \mathrm{~min}$ at room temperature. The supernatant was recentrifuged at $30,000 \mathrm{rpm}$ for $2 \mathrm{hr}$ at room temperature. To precipitate the protein, cold acetone, $-20^{\circ} \mathrm{C}$, was added to the supernatant at a concentration of over $70 \%$, and this was incubated overnight at $-20^{\circ} \mathrm{C}$. Sediments were collected and cold acetone was added (at a final concentration of $70 \%$ ). After centrifugation at 3000-5000 rpm for 5-10 min, pellets were resuspended in $70 \%$ acetone at $0^{\circ} \mathrm{C}$. Finally, the pellets collected by centrifugation at $3000-5000 \mathrm{rpm}$ were dialyzed in $0.01 \mathrm{M}$ PBS and condensed.

To prepare a $\mathrm{McAb}$, we injected the immunogen prepared by the above methods (MAP1 or MT proteins) intraperitoneally or into the footpads of BALB/c mice on 5 occasions. Three days after the final injection, immune lymphocytes from spleen and popliteal lymph nodes were collected and fused (Kohler and Milstein, 1976) with P3-X63-Ag8653 mouse myeloma cells in a solution containing RPMI-1640 (Gibco Laboratories, Grand Island, NY), $40 \%$ polyethylene glycol 2000 , and $20 \%$ dimethyl sulfoxide (DMSO). Cells were plated in 96-well tissue culture dishes, where they were maintained in HAT-selective medium for about a 2 week postfusion. Hybridomas were screened by the ELISA system, using MT proteins as the antigen, and by the immunoblotting method (Towbin et al., 1979). After limiting dilutions and screenings, we produced a single monoclone secreting anti-MAP1 McAb. The immunoreactive protein was identified on the nitrocellulose replica of the SDS gel ( $7 \%$ polyacrylamide) and on that of the SDS/urea gel ( $4 \%$ polyacrylamide) (Bloom et al., 1985) onto which rat brain crude extracts or MT proteins had been blotted.

Immunofluorescence microscopy. Cerebella were dissected from rats perfused with $2 \%$ paraformaldehyde and $0.1 \%$ glutaraldehyde in PEM buffer, pH 6.8 (100 mM piperazine $N, N^{\prime}$-bis[2-ethanesulfonic acid] (PIPES), 1 mм EGTA, $1 \mathrm{~mm} \mathrm{MgCl}_{2}$ ). Fixation was continued for an additional $2 \mathrm{hr}$ in the same fixative at room temperature. Microslicer sections $(40 \mu \mathrm{m})$ were collected in $0.1 \mathrm{M}$ phosphate buffer $(\mathrm{pH} 7.4)$ and processed in suspension for immunofluorescence microscopy. Sections were preincubated overnight with $20 \%$ goat serum in $0.01 \mathrm{~m}$ PBS at $4^{\circ} \mathrm{C}$ in the presence of $0.25 \%$ Triton X-100 to block nonspecific staining, then incubated with undiluted primary antibody (culture medium of

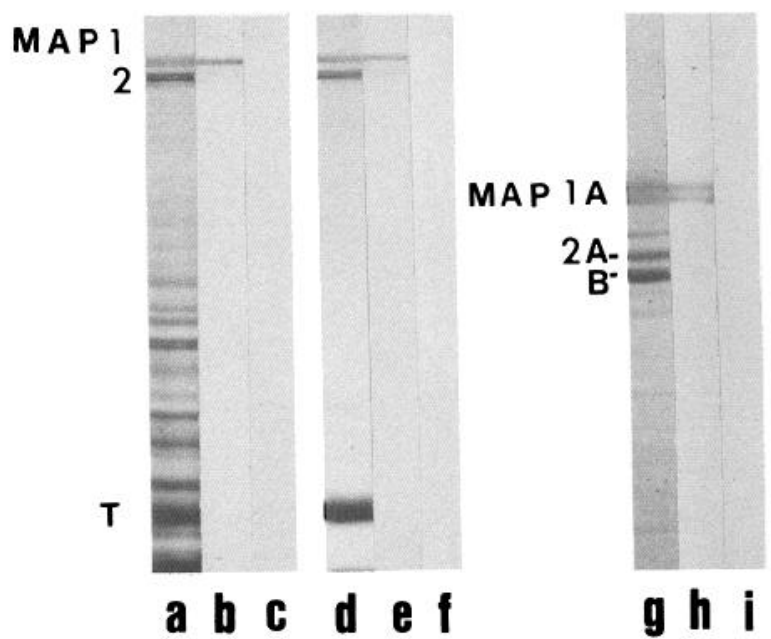

Figure 1. Antigenic specificity of anti-MAP1A McAb. Rat brain crude extracts (lanes $a, b, c$ ) and rat brain microtubule proteins (lanes $d, e, f$ ) were subjected to SDS-PAGE on a 7\% polyacrylamide gel. The positions of $M A P 1, M A P 2$, and tubulin $(T)$ were indicated. When high-molecularweight MAPs were separated in an SDS/urea gel (4\% polyacrylamide), MAP1 and MAP2 were fractionated into MAP1A, 1B, 1C, 2A, and 2B (lanes $g, h, i$ ). Bands of $M A P 1 A, 2 A$, and $2 B$ were indicated. Our McAb showed specific staining against MAP1 (lanes $b, e$ ), and especially against MAP1A (lane $h$ ). Lanes $a, d$, and $g$ were gels stained with Coomassie brilliant blue R250; lanes $b, e$, and $h$ were nitrocellulose replicas stained with anti-MAP1A McAb; lanes $c, f$, and $i$ were nitrocellulose replicas stained with conditioned medium from myeloma cells.

anti-MAP1A McAb or conditioned medium from myeloma cells as control) and with a second antibody (a fluorescein-conjugated IgG fraction of goat anti-mouse IgG) (Cappel Laboratories, Cochranville, PA) containing $0.025 \%$ Triton X-100 for $24-48 \mathrm{hr}$ at $4^{\circ} \mathrm{C}$. Stained sections were washed in PBS and mounted on glass slides with coverslips.

Electron-microscopic immunocytochemistry. The rat cerebellum was dissected out and incubated with $1 \%$ Triton X-100 in the fixative $(2 \%$ paraformaldehyde, $0.1 \%$ glutaraldehyde, $10 \mu \mathrm{M}$ taxol in PEM buffer, $\mathrm{pH} \mathrm{6.8)} \mathrm{for} 40 \mathrm{~min}$ at room temperature. Samples were transferred to the fixative without Triton X-100 and fixed for a total of $2 \mathrm{hr}$. After rinsing with rinse buffer ( $4 \%$ sucrose in $0.1 \mathrm{M}$ phosphate buffer, $\mathrm{pH} 7.4$ ), the tissues were incubated with $1 \% \mathrm{NaBH}_{4}$ in rinse buffer, and then washed again thoroughly in rinse buffer.

Afterwards, the following incubation procedures for immunostaining were performed. The samples were incubated with $20 \%$ goat serum in Tris-buffer saline (TBS) $(20 \mathrm{~mm}$ Tris, $0.9 \% \mathrm{NaCl})$, pH 7.6 , for $3 \mathrm{hr}$ at room temperature to block nonspecific reactions. Incubation with the first antibody (anti-MAP1 McAb or conditioned medium from myeloma cells) was continued for a total of $19 \mathrm{hr}$ at room temperature and/ or at $4^{\circ} \mathrm{C}$. The tissues were washed in TBS, $\mathrm{pH} 8.2$, with $1 \%$ goat serum for $1 \mathrm{hr}$ at room temperature, incubated with the second antibody (goldconjugated goat IgG anti-mouse IgG, 5nm) (Janssen Pharmaceutica, Beerse, Belgium) for a total of $19 \mathrm{hr}$, and then washed again in TBS, $\mathrm{pH} 8.2$, with $1 \%$ goat serum for $1 \mathrm{hr}$. After incubation with the second antibody, the samples were fixed and processed by a conventional procedure for electron microscopy (fixation: $1 \%$ glutaraldehyde in $0.1 \mathrm{M}$ sodium cacodylate buffer, $\mathrm{pH} 7.2 ; 1 \% \mathrm{OsO}_{4}$ in $0.1 \mathrm{M}$ sodium cacodylate buffer, $\mathrm{pH} 7.2$; dehydration, and embedding in Epon 812).

Quick-freeze, deep-etch electron microscopy of Purkinje cell dendrites. In order to observe the 3-dimensional cytoskeletal ultrastructure of dendrites, we prepared fresh and saponin-extracted samples. Slices of rat cerebellum were incubated with $0.1 \%$ saponin in PEM buffer, $\mathrm{pH}$ 6.8 , containing $0.3 \mathrm{~mm}$ phenylmethylsulfonyl fluoride, $1 \mathrm{mg} / \mathrm{ml} \mathrm{leu}-$ peptin, and $10 \mu \mathrm{M}$ taxol, for $30 \mathrm{~min}$ at room temperature (Hirokawa, 1982). Both fresh and saponin-extracted tissues were quick-frozen, freezefractured, deep-etched, and rotary-shadowed with platinum and carbon, as described previously (Heuser and Salpeter, 1979; Hirokawa and Heuser, 1981).

Purification of MAP1A. MAP1A was purified by a CNBr-activated Sepharose 4B column using McAb against MAP1A. After application of the brain crude extracts, the column was washed with $0.01 \mathrm{M}$ phosphate buffer containing $0.75 \mathrm{M} \mathrm{NaCl}$ and $0.5 \%$ Nonidet P-40. Bounded 


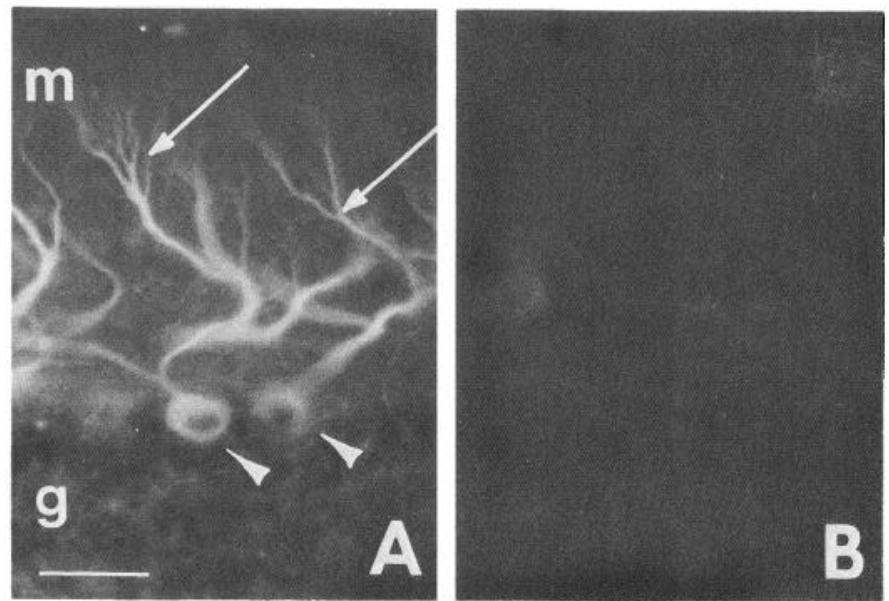

Figure 2. Immunofluorescence microscopy of sagittal sections of a cerebellar cortex. Sections shown in $A$ and $B$ were taken from the same tissue block. $A$, Anti-MAP1A McAb stained Purkinje cell perikarya and dendrites brightly. $m$, Molecular layer; $g$, granule cell layer; arrowheads, Purkinje cell body; arrows, Purkinje cell dendrite. Bar, $40 \mu \mathrm{m}$. B, Control section stained with conditioned medium from myeloma cells, showing no staining.

polypeptides were eluted with $3 \mathrm{M} \mathrm{MgCl}_{2}$ in 0.1 M PEM buffer, peak fractions dialyzed in $20 \mathrm{mM}$ PEM buffer, and were then subjected to PAGE ( $7 \%$ polyacrylamide and $4 \%$ SDS/urea polyacrylamide) to check their purity.
Table 1. Distribution of gold particles on the dendritic cytoskeletal elements shown by immunoelectron microscopy

Marked sites

Gold particles (\%)

On MTs

On fuzzy materials associated with MTs or between MT and MT

Associated with NFs

Associated with MOs

On other structures

5.2
Structures of MAPIA and taxol-stabilized MTs decorated with MAPIA. The molecular structure of MAP1A was revealed by the rotary shadowing method (Tyler and Branton, 1980). The solution of MAP1A with glycerol (at a final concentration of $60 \%$ ) was sprayed onto pieces of freshly cleaved mica. Soon after, they were dried in vacuo at room temperature for $1-4 \mathrm{hr}$, rotary-shadowed with platinum $(2.5 \mathrm{~nm})$ at an angle of $6^{\circ}$ and then overcoated with carbon.

The structure of taxol-stabilized MT pellets composed of tubulin and MAP1A was studied by QF-DE. MTs decorated with MAP1A were prepared by the following method. Microtubule proteins from rat brains were purified by 3 cycles of temperature-dependent assembly and disassembly in PEM buffer, as described by Shelanski et al. (1973). Tubulin was isolated from microtubule proteins by phosphocellulose chromatography under cold conditions, as described by Herzog and Weber (1978). Pure tubulin was eluted with PEM buffer. The purified tubulin

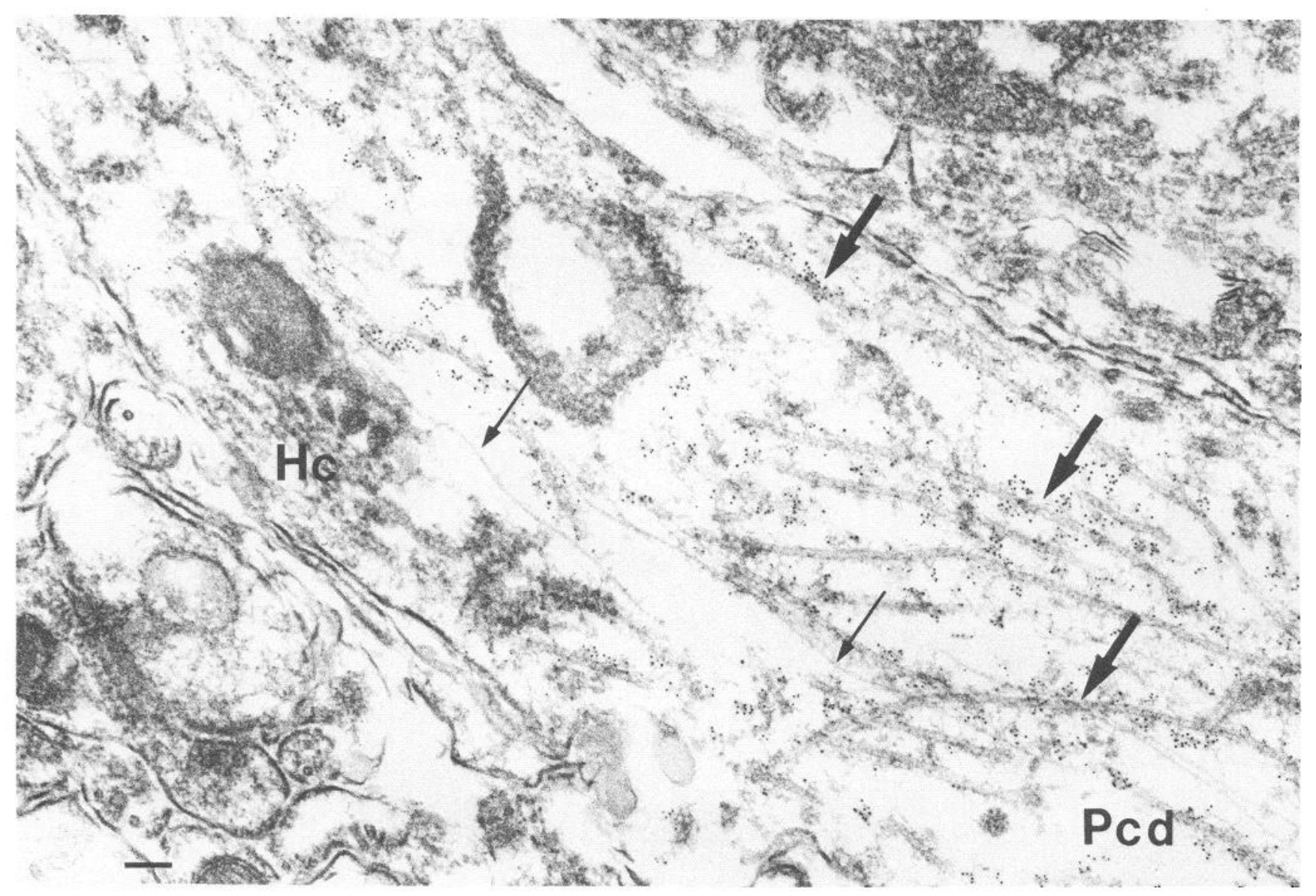

Figure 3. Immunoelectron microscopy of a Purkinje cell dendrite stained with anti-MAP1A McAb followed by immunogold staining. Fuzzy materials along with dendritic MTs were specifically stained with anti-MAP1A McAb. Structures in adjacent synapses and fuzzy materials, along with dendritic NFs, were not stained. $P c d$, Purkinje cell dendrite; large arrows, microtubule; small arrows, neurofilament; $H c$, hypolemmal cisternae. $\times 52,000$. Bar, $0.2 \mu \mathrm{m}$. 


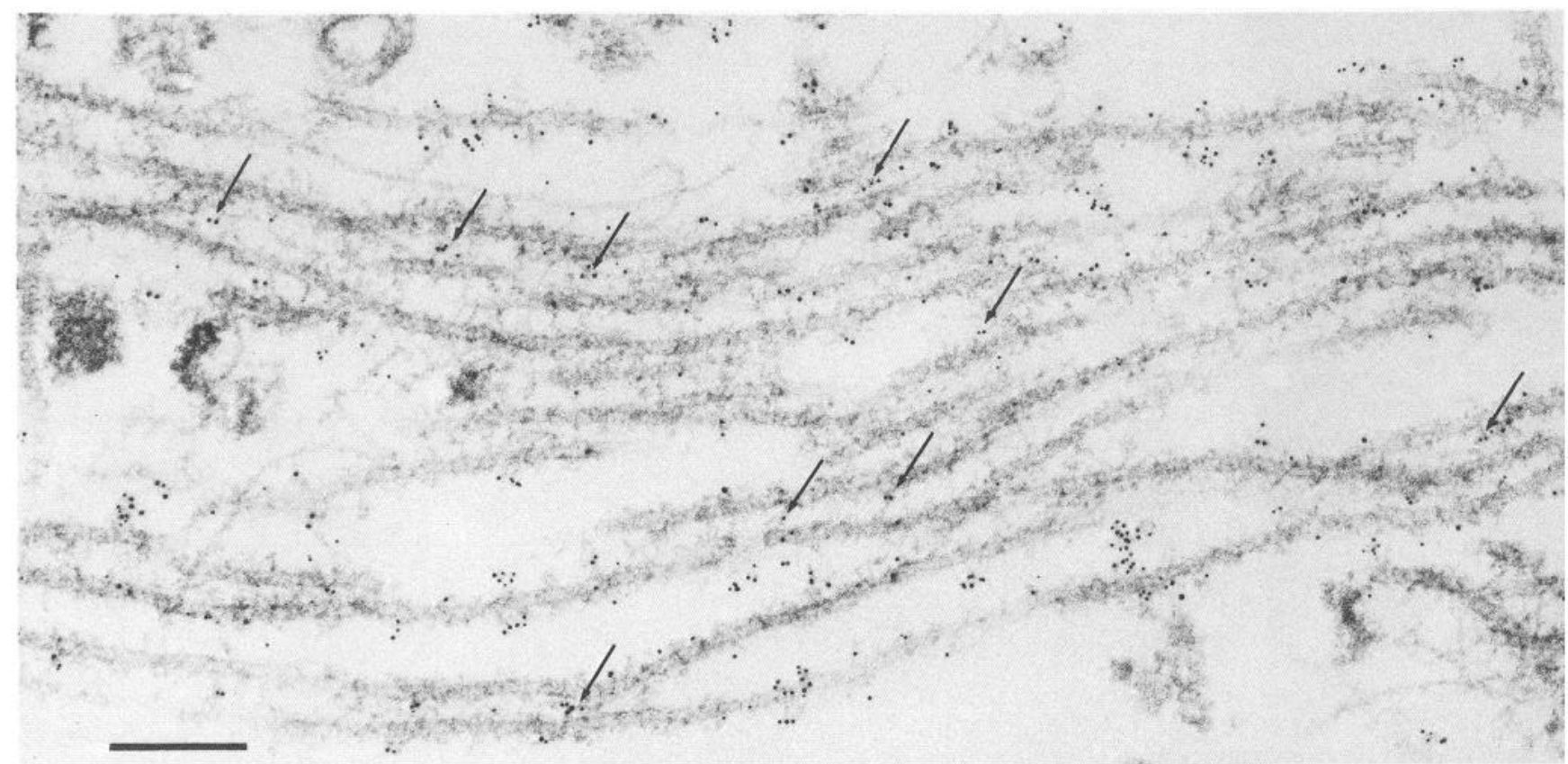

Figure 4. Higher magnification of a dendrite. MTs were connected to one another by filamentous projections or fuzzy materials (thin arrows) on which gold particles were specifically localized. $\times 76,000$. Bar, $0.2 \mu \mathrm{m}$.

was incubated with affinity-purified MAP1A (at a quantitative ratio of $1: 1$ to $8: 1$ ) in PEM buffer at $37^{\circ} \mathrm{C}$ for $10 \mathrm{~min}$ by the addition of $1 \mathrm{~mm}$ GTP and $20 \mu \mathrm{M}$ taxol. After centrifugation at $38,000 \mathrm{rpm}$ at $30^{\circ} \mathrm{C}$ for $30 \mathrm{~min}$, the pellets were processed for QF-DE.

Thin sections and replicas were observed using a JEOL 1200EX EM at $100 \mathrm{kV}$.

\section{Results}

Anti-MAPIA monoclonal antibody

When the blotted channels of unfractionated rat brain crude extracts and MT proteins obtained by the taxol method were stained with our McAb using an immunoperoxidase procedure, bands corresponding to MAP1 (7\% polyacrylamide; Fig. 1,b,e) and MAP1A (SDS/urea, 4\% polyacrylamide; Fig. $1 h$ ) were specifically stained. On the other hand, condítioned medium from myeloma cells used as a control showed no staining (Fig. 1, $c, f, i$ ).

While Bloom et al. (1984) reported that MAP1 of MT proteins from calf brain white matter was separated into 3 bands of $1 \mathrm{~A}$, $\mathrm{IB}$, and $\mathrm{IC}$, we found that MAP1A obtained from adult rat whole brain was separated into 2 bands, and that the band of MAP1B was hardly detectable, as shown in Fig. $1 \mathrm{~g}$. Our McAb against MAP1 reacted with 2 bands of MAP1A (Fig. $1 h$ ).

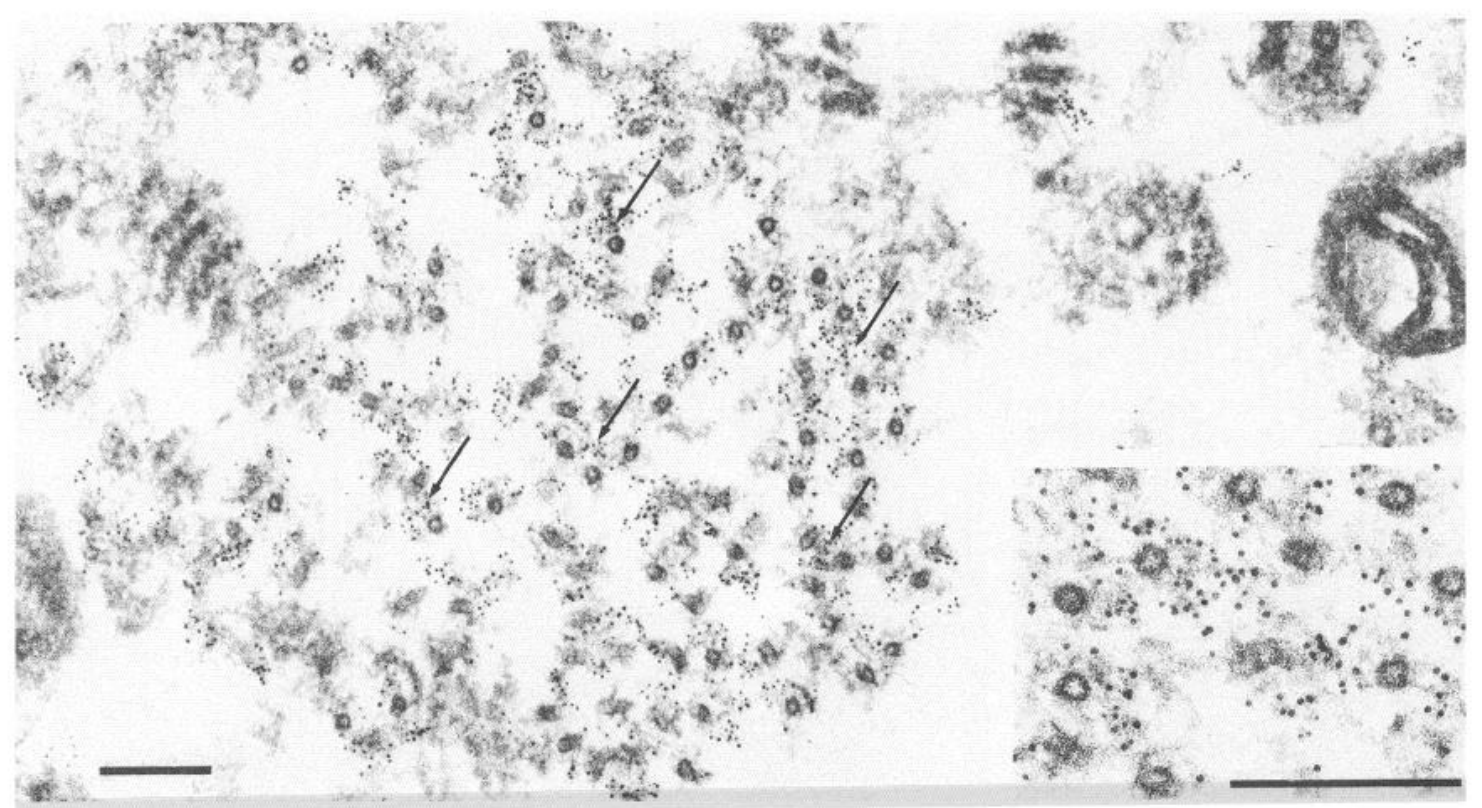

Figure 5. Immunoelectron microscopy of a cross section of a dendrite. Gold particles were studded on fuzzy materials that projected from MTs. Most of these structures (arrows) cross-linked MTs to one another. $\times 57,000$. Inset, Higher-magnification of a cross section. Immunogolds located on filamentous projections that connect MTs to one another. $\times 118,000$. Bars, $0.2 \mu \mathrm{m}$. 


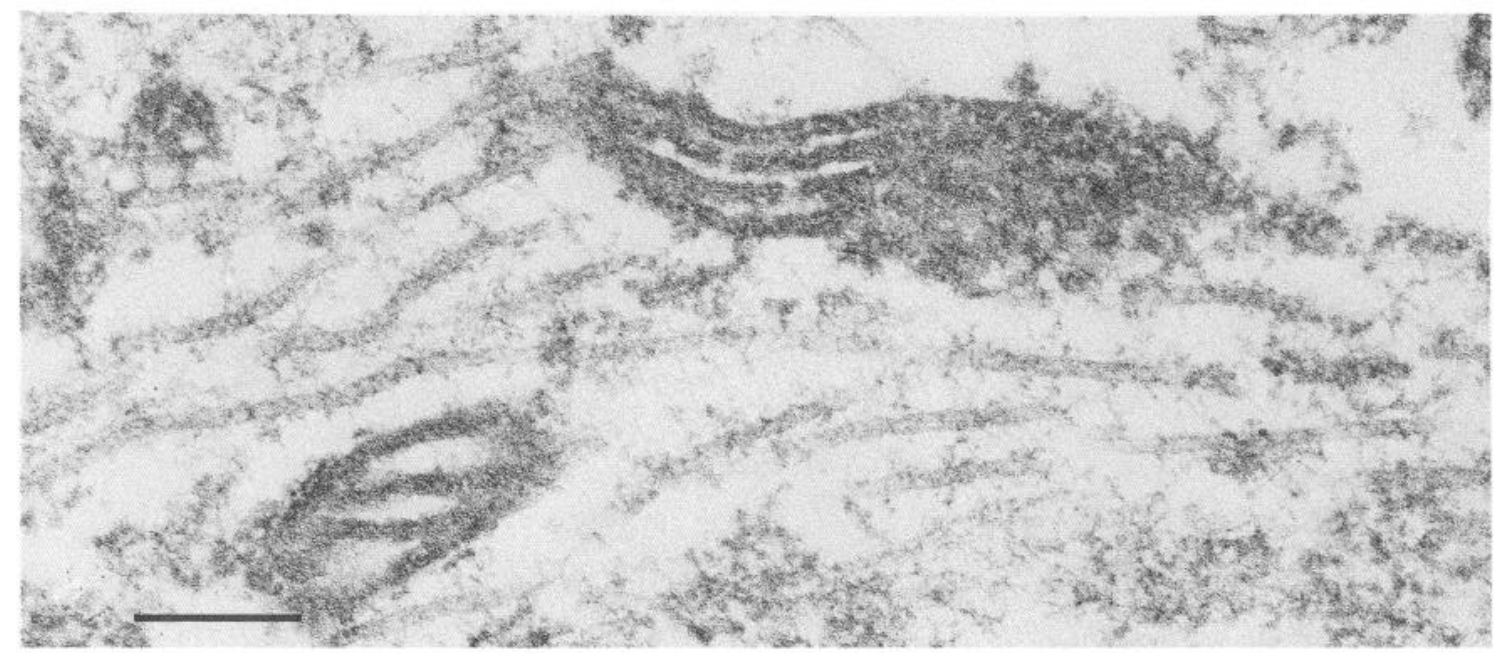

Figure 6. Immunoelectron microscopy of a control section for anti-MAP1A McAb staining. It shows no staining. $\times 76,000$. Bar, $0.2 \mu \mathrm{m}$.

\section{Immunofluorescence microscopy}

Our anti-MAP1A McAb was examined immunofluorescently on the rat cerebellar cortex. Purkinje cell perikarya and dendrites were always stained brightly (Fig. $2 A$ ) and, in some sections, we found Purkinje cell axons stained in the granule layer. These results coincided well with those of previous studies using antiMAP1 antibodies (Matus et al., 1983; Bloom et al., 1984; Huber and Matus, 1984).

\section{Electron-microscopic immunocytochemistry}

To localize MAP1A within the dendritic cytoskeletons, we prepared slices of rat cerebellum extracted with Triton X-100 and labeled them with monoclonal anti-MAP1A, followed with immunogold staining. Figure 3 shows a Purkinje cell dendrite labeled with anti-MAP1A antibody. Purkinje cell dendrites were identified by the presence of the characteristic hypolemmal cisternae and postsynaptic thorns. Gold particles were found specifically within the dendrite, whereas we could hardly observe any gold particles in adjacent synapses. The particles were studded on the filamentous fuzzy materials attached to MTs or connecting MTs to one another, but they were not observed on those associated with NFs. We also found a few particles on the material between MTs and membrane organelles such as mitochondoria. Electron micrographs of a higher magnification (Fig. 4) and a cross section of a dendrite (Fig. 5) display more clearly the localization of most gold particles on the projecting portions of filamentous fuzzy materials between MTs. The inset in Figure 5 shows such filamentous structures connecting MTs to one another. The distribution of these gold particles within dendrites is shown in Table 1. The data also indicated clearly that most of the gold particles labeled MT-associated cytoskeletal materials. Samples incubated with a control medium showed no staining (Fig. 6).

\section{Quick-freeze, deep-etch electron microscopy}

Dendritic cytoskeletons were observed by QF-DE. Figure 7 presents a low-magnification view of a Purkinje cell dendrite in a fresh cerebellum. MTs were predominantly found in dendrites, with only a few NFs. Striking features revealed by QF-DE in the dendrites were the elaborate networks of cross-bridges between MTs. These cross-bridges, branching and anastomosing much more extensively than those in axons, not only linked MTs to one another, but also connected membrane organelles (MOs), or plasmalemma to MTs. Furthermore, this network of MT-associated cross-bridges completely filled the cytoplasm in the dendrites, in contrast to the axon. The axonal cytoskeleton was mainly composed of numerous neurofilament lattices and sparsely distributed microtubule bundles (Fig. 8). The overall image of the anastomosing networks between MTs in the dendrites was sometimes granular in appearance, partly because of the attachment of globular materials. MTs were characterized by longitudinal arrays of protofilaments. Bumps were seen sporadically on the surface.

A higher-magnification view of a fresh dendrite (Fig. 9A) highlight the detailed structures of cross-bridges between MTs. Hypolemmal cisternae and endoplasmic reticulum were also cross-linked to MTs by filamentous, anastomosing cross-bridges. To circumvent the possibility that these structures were artifactual results of condensations or precipitations of soluble proteins during deep etching, we examined saponin-treated tissues from which soluble proteins had been extracted. As can be seen in Figure $9 B$, networks of cross-bridges were preserved in the saponin-extracted dendrite. Though the filamentous appearance of the cross-bridges was more pronounced than that of the fresh samples, the width of the cross-bridges was still sometimes irregular.

\section{Purification of MAP1A}

In order to match the results of immunoelectron microscopy with the cytoskeletal structures observed by QF-DE, we further investigated the molecular structure and the appearance of MAP1A while attached to MTs in vitro. The purity of affinitypurified MAP1A is shown in Figure 10A. The results of PAGE indicated that only MAP1A was predominant in the fraction (Fig. 10, $A, d, g$ ).

\section{Structures of MAPIA and taxol-stabilized MTs decorated with MAPIA}

The molecular structure of MAP1A obtained by the rotaryshadowing method revealed that several individual molecules were long, thin, filamentous structures (ranging from about 100 to $150 \mathrm{~nm}$ in length) possessing considerable flexibility (Fig. $10 B$ ). Some molecules extended only slightly, and others ap- 


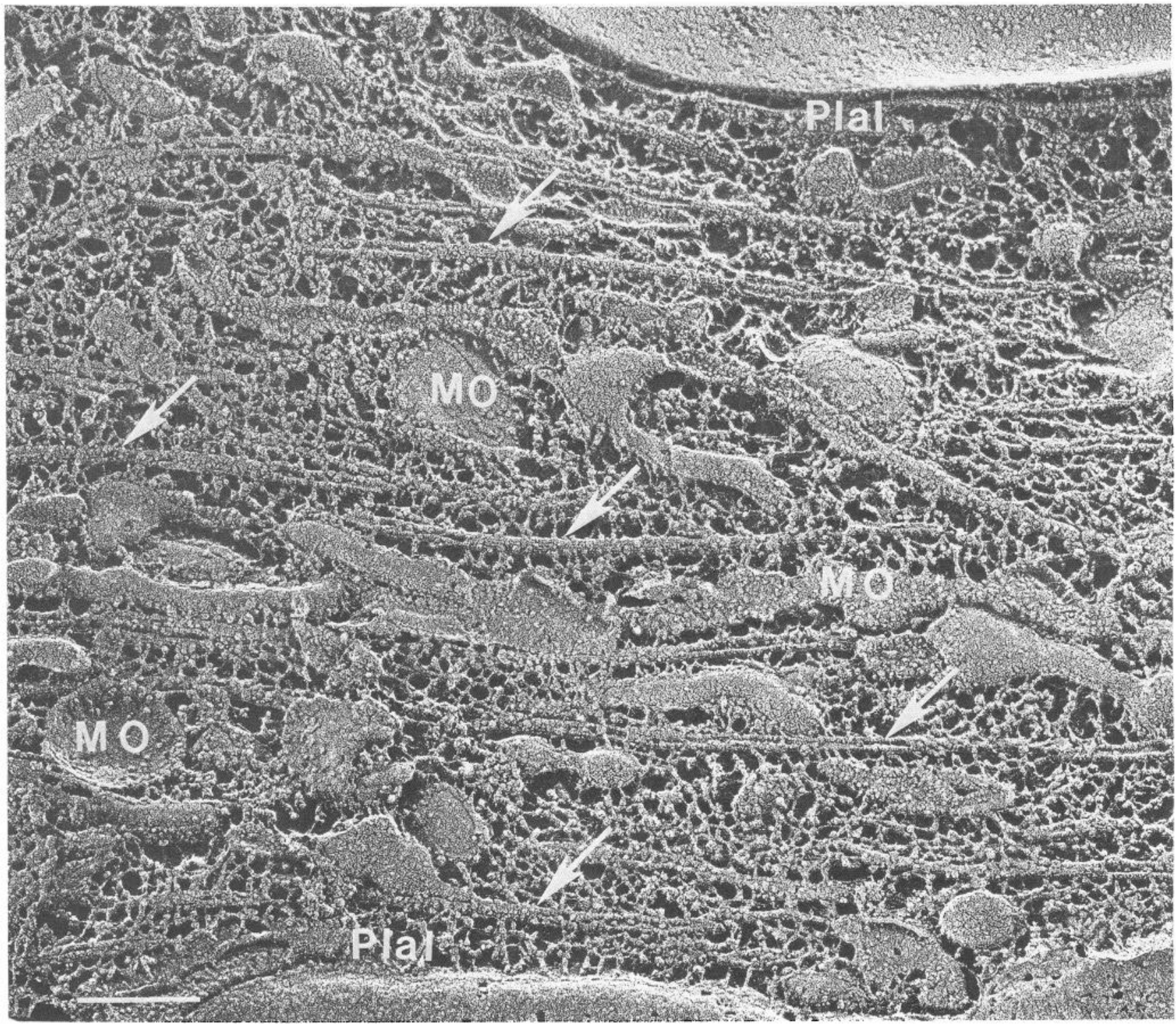

Figure 7. Low-magnification view of a quick-frozen, deep-etched Purkinje cell dendrite in a fresh cerebellum. MTs (large arrows) were predominantly found in a dendrite. MTs were linked to one another by elaborate networks of cross-bridges that sometimes branched and anastomosed. In addition, MTs were connected to MOs, NFs, or plasmalemma by the cross-bridges, which were somewhat granular in appearance. $M O$, Membrane organelle; Plal, plasmalemma. $\times 91,000$. Bar, $0.2 \mu \mathrm{m}$.

peared to be shorter filaments that may perhaps have been only fragments of molecules (data not shown). MTs assembled in the presence of taxol with purified MAP1A were observed by QFDE. As demonstrated in Figure 11, many thin, filamentous crossbridges, presumably MAP1A molecules, cross-linked the MTs to one another. These samples were subjected to PAGE to insure that they contained only tubulin and MAP1A (Fig. 10,A,e). Thin, filamentous cross-bridges were not present on the MTs from which MAPs were extracted by high-salt treatment (data not shown; see the earlier report of Hirokawa et al., 1985).

\section{Discussion}

We have demonstrated the single-molecular structure of MAP1A and clearly displayed its distribution in dendritic cytoskeletons at the molecular level for the first time. By immunoelectron microscopy, we have shown that fuzzy materials between MTs in Purkinje cell dendrites were heavily marked by our anti-
MAP1A McAb (Figs. 3-5). The ultrastructural images observed by QF-DE revealed networks of extensive, elaborate cross-bridges within dendrites (Fig. 7). To juxtapose the results of immunoelectron microscopy with the cytoskeletal structures observed by QF-DE, we performed a number of experiments in vitro. We examined the structures of the affinity-purified MAP1A molecule and of MTs decorated with MAP1A. The MAP1A molecule took a long, thin, filamentous, and very flexible shape and formed filamentous cross-bridges between MTs when MAP1A and tubulin were incubated in the presence of taxol. These in vitro results indicated that MAP1A could be responsible for the presence of both fuzzy materials and cross-bridges in vivo. Consequently, we concluded that MAP1A was one of the major components of the elaborate network of cross-bridges between microtubules in Purkinje cell dendrites.

There are regional differences in the structures and chemical composition of neurons. In dendrites, abundant MTs are dis- 


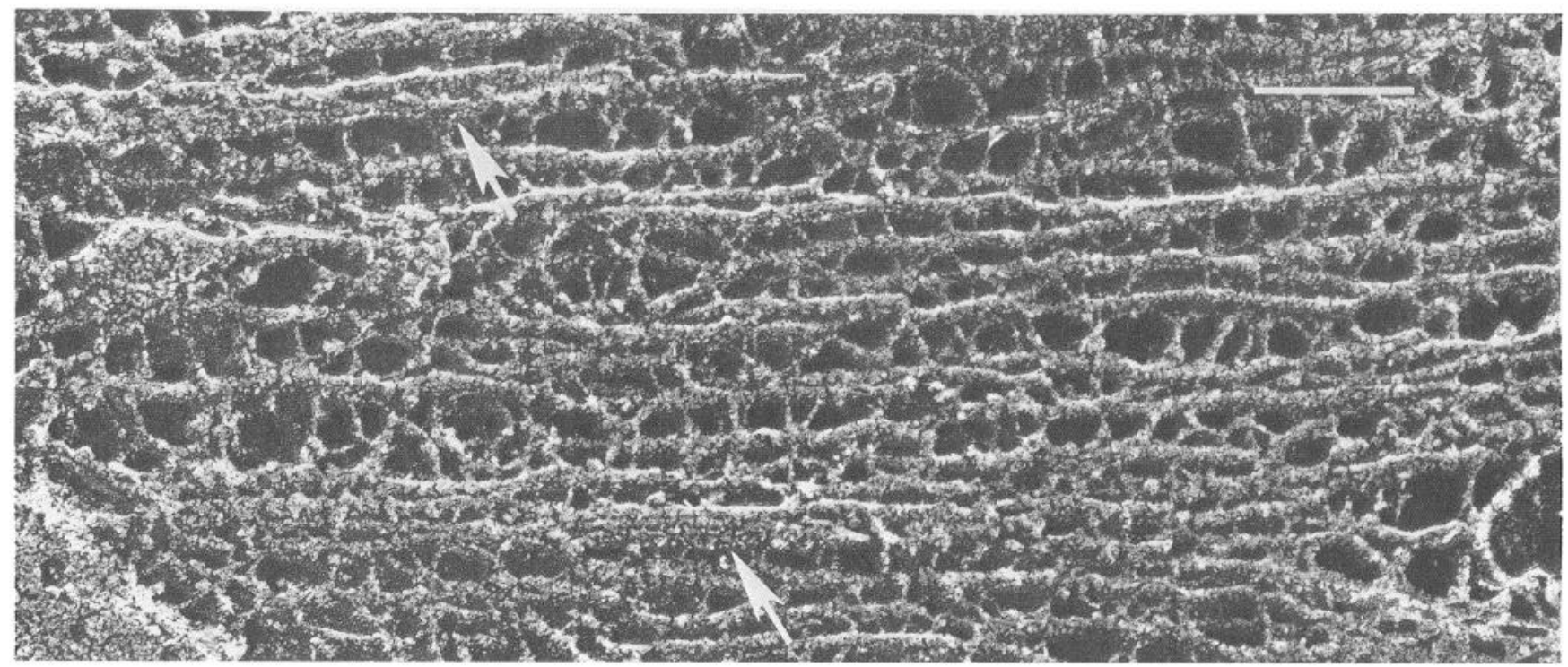

Figure 8. Axonal cytoskeleton observed in the molecular layer of the rat cerebellum. Microtubules (arrows) were sparcely distributed within numerous neurofilament lattices. $\times 179,000$. Bar, $0.1 \mu \mathrm{m}$.

tributed uniformly, with few neurofilaments. Axonal MTs, on the other hand, are generally organized into a MT domain of small, tightly packed clusters within abundant neurofilaments (Hirokawa, 1982). QF-DE, in this report, revealed the tremendously elaborate network of cross-bridges that developed throughout the dendrites, filling up the spaces among MTs, and between MTs and MOs, and plasmalemma. MT-associated cross- bridges in dendrites branched and anastomosed more extensively than those observed in axons, although some of their features were somewhat similar to those in the MT domain of IDPN-intoxicated rat axons (Hirokawa et al., 1985).

Immunological (Miller et al., 1982; Matus et al., 1983; Bloom et al., 1984; DeCamilli et al., 1984; Peng et al., 1986) and biochemical (Vallee, 1982; Peng et al., 1986) studies have revealed
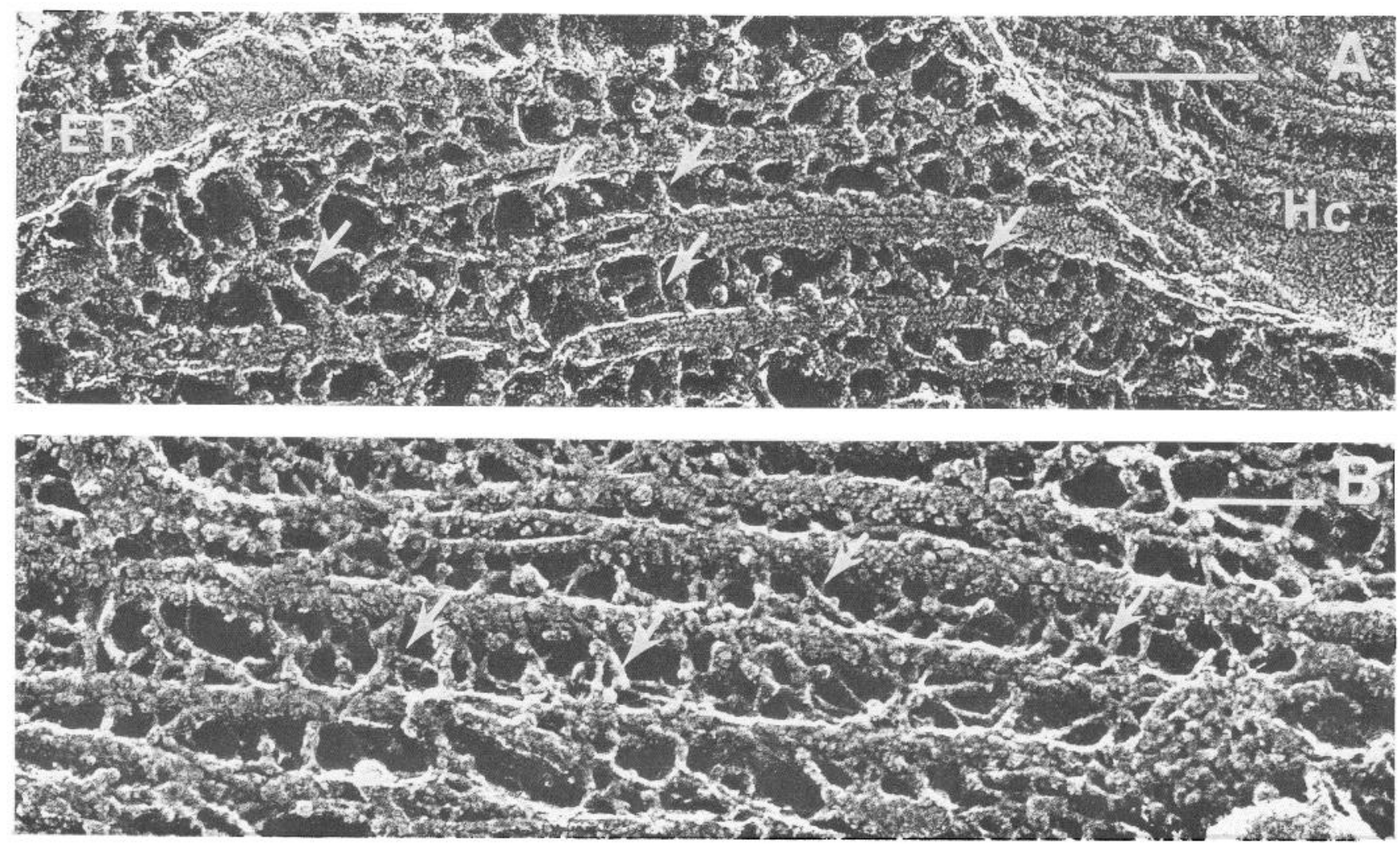

Figure 9. A, Higher magnification of a fresh Purkinje cell dendrite. Cross-bridges (arrows) connected MTs to one another. Hc, Hypolemmal cisternae; $E R$, endoplasmic reticulum. $\times 190,000 . B$, High magnification of a saponin-extracted dendrite. After extraction by saponin, the structure of cross-bridges (arrow) was well preserved and their width was sometimes irregular. $\times 165,000$. Bars $(A, B), 0.1 \mu \mathrm{m}$. 

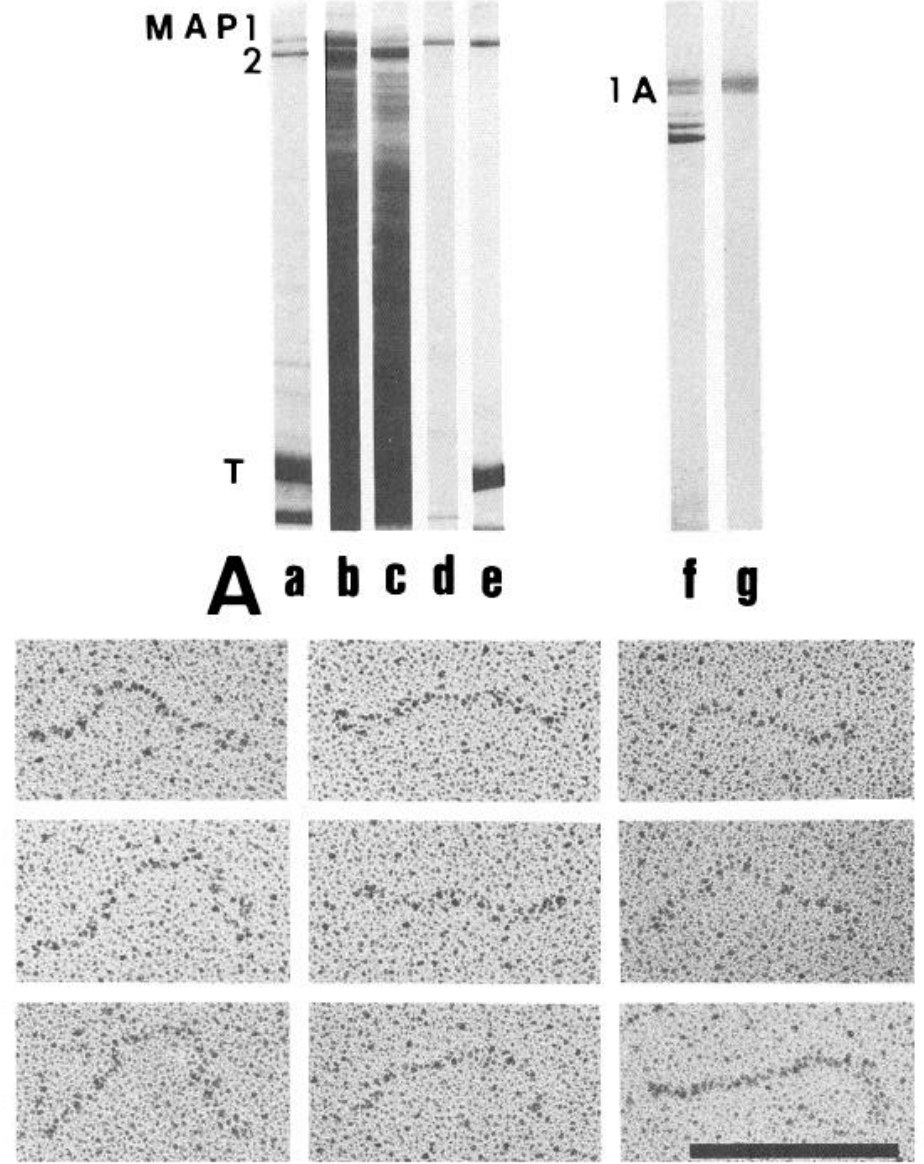

\section{B}

Figure 10. A, MAP1A was purified by the affinity column with high efficiency. It was checked by PAGE on $7 \%$ polyacrylamide gels (lanes $a, b, c, d, e$ ) and by $4 \%$ SDS/urea polyacrylamide gels (lanes $f, g$ ). $a$, Rat brain MT proteins obtained by taxol method (MAP1, $M A P 2$, and tubulin, $T$, were indicated); $b$, rat brain crude extracts that were applied to the affinity column; $c$, flowthrough (note that the amount of MAPIA is markedly decreased compared to the crude extracts); $d$, the peak fraction of MAP1A eluted by $3 \mathrm{M} \mathrm{MgCl}_{2}$ (in this fraction, only MAP1 was eluted); $e$, taxol-stabilized MT pellets decorated with affinity-purified MAP1A; $f$, MT proteins separated into MAP1A, 1B, 1C, 2A, and 2B (bands corresponding to $M A P 1 A$ are indicated); $g$, affinity-purified MAP1A. This gel indicated that the MAP1A was clearly purified. $B$, Molecular structures of MAP1A obtained by the rotary-shadowing method. Molecules have extended long, thin, flexible structures. $\times 214,000$. Bar, $100 \mathrm{~nm}$. the regional differences in the chemical components of neurons. For example, MAP1 exists equally in both axons and dendrites (Matus et al., 1983; Bloom et al., 1984; Huber and Matus, 1984; Peng et al., 1986); MAP2 is abundant in dendrites but scarcely exists in axons (Vallee, 1982; Papasozomenos et al., 1985); and tau is prominent in axons (Binder et al., 1984; Peng et al., 1986). The regional differences in composition are likely responsible for the corresponding differences in structure.

We considered the possibility that MAP2, at least, was another component of the MT-associated cross-bridges in dendrites, because many investigators have reported that purified MAP2, a long, rodlike molecule (Voter and Erikson, 1982), forms filamentous arms on the surface of MTs in vitro (Herzog and Weber, 1978; Kim et al., 1979; Gottlieb and Murphy, 1983, 1985). Probably MAP1 and MAP2 have a structural interrelationship within the network of dendritic cross-bridges. This may explain the more complicated features of cross-bridges in dendrites than in axons.

There may be yet other molecules besides MAP1A and MAP2, such as those possibly responsible for the granular appearance of the cross-bridges observed in this study, and all of these molecules are presumed to be involved in the interactions of MTs with one another and with cellular components. In order to further clarify the functions of MTs and MAPs in dendrites in vivo, additional studies directed at the identification of each of the molecular components of the cross-bridges between MTs and MTs and with other cellular components need to be carried out.

\section{References}

Binder, L. I., A. Frankfurter, and L. I. Rebhun (1984) A monoclonal antibody to tau-factor localizes predominantly in axons. J. Cell Biol. (Abstr.) 99: 191a.

Bloom, G. S., and R. B. Vallee (1983) Association of microtubuleassociated protein 2 (MAP2) with microtubules and intermediate filaments in cultured brain cells. J. Cell Biol. 96: 1523-1531.

Bloom, G. S., T. A. Schoenfeld, and R. B. Vallee (1984) Widespread distribution of the major polypeptide component of MAP1 (microtubule-associated protein 1) in the nervous system. J. Cell Biol. 98 . 320-330.

Bloom, G. S., F. C. Luca, and R. B. Vallee (1985) Microtubule-associated protein 1B: A novel, major component of the neuronal cytoskeleton. Proc. Natl. Acad. Sci. USA 82: 5404-5408.

Borisy, G. G., J. M. Marcum, J. B. Olmsted, D. B. Murphy, and K. A. Johnson (1975) Purification of tubulin and associated high molecular weight proteins from porcine brain and characterization of microtubule assembly in vitro. Ann. NY Acad. Sci. 253: 107-132.
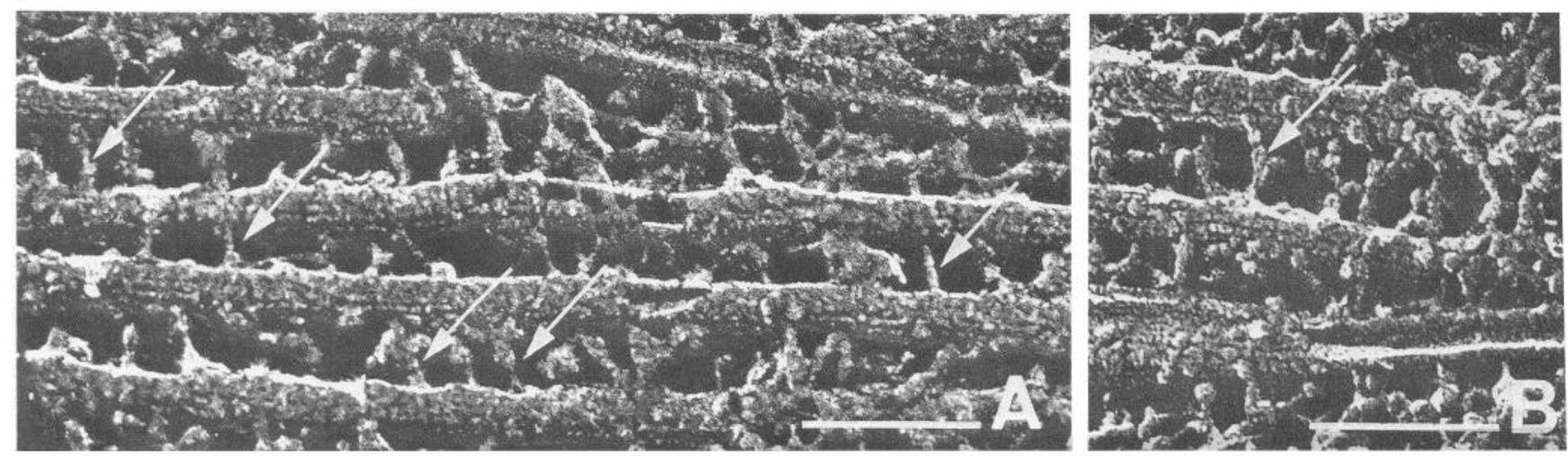

Figure 11. Ultrastructure of taxol-stabilized MTs decorated with MAP1A processed by QF-DE. Many thin, filamentous cross-bridges (arrows) connect MTs to one another. $A, \times 199,000 . B, \times 216,000$. Bars $(A, B), 0.1 \mu \mathrm{m}$. 
Cleveland, D. W., S. Hwo, and M. W. Kirschner (1977a) Purification of tau, a microtubule-associated protein that induces assembly of microtubules from purified tubulin. J. Mol. Biol. 116: 207-225.

Cleveland, D. W., S. Hwo, and M. W. Kirschner (1977b) Physical and chemical properties of purified tau factor and the role of tau in microtubule assembly. J. Mol. Biol. 116: 227-247.

DeCamilli, P., P. E. Miller, F. Navone, W. E. Theurkauf, and R. B. Vallee (1984) Distribution of microtubule-associated protein 2 in the nervous system of the rat studied by immunofluorescence. Neuroscience 11: 819-846.

Gottlieb, R. A., and D. B. Murphy (1983) The pattern of MAP-2 binding on microtubules: Visual enhancement of MAP attachment sites by antibody labeling and electron microscopy. J. Ultrastruct. Res. 85: 175-185.

Gottlieb, R. A., and D. B. Murphy (1985) Analysis of the microlubulebinding domain of MAP-2. J. Cell Biol. 101: 1782-1789.

Herzog, W., and K. Weber (1978) Fractionation of brain microtubuleassociated proteins (isolation of two different proteins which stimulate tubulin polymerization in vitro). Eur. J. Biochem. 92: 1-8.

Heuser, J. E., and S. R. Salpeter (1979) Organization of acetylcholine receptors in quick-frozen, deep-etched, and rotary-replicated Torpedo postsynaptic membrane. J. Cell Biol. 82: 150-173.

Hirokawa, N. (1982) Cross-linker system hetween neurofilaments, microtubules, and membranous organelles in frog axons revealed by the quick-freeze, deep-etching method. J. Cell Biol. 94: 129-142.

Hirokawa, N., and J. E. Heuser (1981) Quick-freeze, deep-etch visualization of the cytoskeleton beneath surface differentiations of intestinal epithelial cells. J. Cell Biol. 91: 399-409.

Hirokawa, N., and H. Yorifuji (1986) Cytoskeletal architecture of reactivated crayfish axons, with special reference to crossbridges among microtubules and between microtubules and membrane organelles. Cell Motil. 6: 458-468.

Hirokawa, N., M. A. Glicksman, and M. B. Willard (1984) Organization of mammalian neurofilament polypeptides within the neuronal cytoskeleton. J. Cell Biol. 98: 1523-1536.

Hirokawa, N., G. S. Bloom, and R. B. Vallee (1985) Cytoskeletal architecture and immunocytochemical localization of microtubuleassociated proteins in regions of axons associated with rapid axonal transport: The $\beta, \beta^{\prime}$-iminodipropionitrile-intoxicated axon as a model system. J. Cell Biol. 101: 227-239.

Huber, G., and A. Matus (1984) Immunocytochemical localization of microtubule-associated protein 1 in rat cerebellum using monoclonal antibodies. J. Cell Biol. 98: 777-781.

Kim, H., L. I. Binder, and J. L. Rosenbaum (1979) The periodic association of MAP2 with brain microtubules in vitro. J. Cell Biol. 80: 266-276.
Kohler, G., and C. Milstein (1976) Derivation of specific antibodyproducing tissue culture and tumor lines by cell fusion. Eur. J. Immunol. 6: 511-519.

Laemmli, U. K. (1970) Cleavage of structural proteins during the assembly of the head of bacteriophage T4. Nature 227: 680-685.

Matus, A., G. Huber, and R. Bernhardt (1983) Neuronal microdifferentiation. Cold Spring Harbor Symp. Quant. Biol. 48: 775-782.

Miller, P., U. Walter, W. E. Theurkauf, R. B. Vallee and P. DeCamilli (1982) Frozen tissue sections as an experimental system to reveal specific binding sites for the regulatory subunit of type II cAMPdependent protein kinase in neurons. Proc. Natl. Acad. Sci. USA 79: $5562-5566$.

Papasozomenos, S. C., L. I. Binder, P. K. Bender, and M. R. Payne (1985) Microtubule-associated protein 2 within axons of spinal motor neurons: Associations with microtubules and neurofilaments in normal and $\beta, \beta^{\prime}$-iminodipropionitrile-treated axons. J. Cell Biol. 100 , $74-85$.

Peng, I., L. I. Binder, and M. M. Black (1986) Biochemical and immunological analyses of cytoskeletal domains of neurons. J. Cell Biol. 102: 252-262.

Schnapp, B. J., and T. S. Reese (1982) Cytoplasmic structure in rapidfrozen axons. J. Cell Biol. 94: 667-679.

Shelanski, M. L., F. Gaskin, and C. R. Cantor (1973) Microtubule assembly in the absence of added nucleotide. Proc. Natl. Acad. Sci. USA 70: 765-768.

Sloboda, R. D., S. A. Rudolph, J. L. Rosenbaum, and P. Greengard (1975) Cyclic AMP-dependent endogenous phosphorylation of a microtubule-associated protein. Proc. Natl. Acad. Sci. USA 72: $177-$ 181.

Towbin, H., T. Staehelin, and J. Gordon (1979) Electrophoretic transfer of proteins from polyacrylamide gels to nitrocellulose sheets: Procedure and some applications. Proc. Natl. Acad. Sci. USA 76: 43504354.

Tyler, J. M., and D. Branton (1980) Rotary shadowing of extended molecules dried from glycerol. J. Ultrastruct. Res. 71: 95-102.

Vallee, R. B. (1982) A taxol-dependent procedure for the isolation of microtubules and microtubule-associated proteins (MAPs). I. Cell Biol. 92: 435-442.

Vallee, R. B., and S. E. Davis (1983) Low molecular weight microtubule-associated proteins are light chains of microtubule-associated protein 1 (MAP1). Proc. Natl. Acad. Sci. USA 80: 1342-1346.

Voter, W. A., and H. P. Erikson (1982) Electron microscopy of MAP2 (microtubule-associated protein 2). J. Ultrastruct. Res. 80: 374-382.

Weingarten, M. D., A. H. Lockwood, S. Hwo, and M. W. Kirschner (1975) A protein factor essential for microtubule assembly. Proc. Natl. Acad. Sci. USA 72: 1858-1862. 